DAMTP-2003-12

hep-th/0302072

\title{
Compactification, topology change and surgery theory
}

\author{
Sean A. Hartnoll \\ s.a.hartnoll@damtp.cam.ac.uk \\ DAMTP, Centre for Mathematical Sciences, Cambridge University \\ Wilberforce Road, Cambridge CBз OWA, UK
}

\begin{abstract}
We study the process of compactification as a topology change. It is shown how the mediating spacetime topology, or cobordism, may be simplified through surgery. Within the causal Lorentzian approach to quantum gravity, it is shown that any topology change in dimensions $\geq 5$ may be achieved via a causally continuous cobordism. This extends the known result for 4 dimensions. Therefore, there is no selection rule for compactification at the level of causal continuity. Theorems from surgery theory and handle theory are seen to be very relevant for understanding topology change in higher dimensions. Compactification via parallelisable cobordisms is particularly amenable to study with these tools.
\end{abstract}




\section{Contents}

1 Introduction 1

2 Mathematical preliminaries 3

2.1 Results from relative homology and homotopy theory . . . . . . . . . . . 3

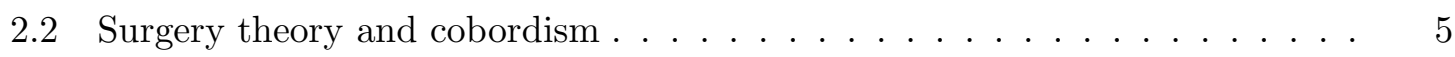

2.3 Handle decomposition, Morse theory and causal continuity . . . . . . . . . 6

3 Quantum compactification: generic case $\quad 8$

3.1 Eliminating the fundamental group . . . . . . . . . . . . . 8

3.2 A causally continuous Morse metric for compactification . . . . . . . . . . 9

4 Compactification via parallelisable cobordisms $\quad 11$

4.1 Eliminating higher homotopy groups . . . . . . . . . . . . . . . . . . 11

4.2 Example: Torus compactification from six to four dimensions . . . . . . . . 13

4.3 Example: $S^{4} \times T^{3}$ compactification from eleven to four dimensions . . . . . 13

5 Completeness of surgery for compactification $\quad 14$

6 Conclusions and discussion $\quad 16$

$\begin{array}{ll}\text { A Surgery as a canonical modification } & 17\end{array}$

\section{Introduction}

The requirement of extra spacetime dimensions is a persistent theme in theories of unification and of quantum gravity. For consistency with experience, the theory must contain a mechanism that would allow only four dimensions to have been observed thus far. Compactification of extra dimensions is thought to be one such mechanism. In this paper we study the topology involved in the process of compactification [1].

By a compactified universe, we mean a universe with spacelike topology $S^{d-1} \times A$. The

sphere $S^{d-1}$ is thought of as the large, observed, space directions and the internal space $A$, of dimension $n-d$, is small and unobserved. Note $n$ is the total dimension of spacetime and $d$ is the observed dimension of spacetime. A universe with spacelike topology $S^{n-1}$ is 'not compactified' and all dimensions are large. 
It seems reasonable to think that, in some regime at least, quantum gravity may be conceived as a path integral over spacetimes [2]. One considers all topologies joining the given initial and final spacelike hypersurfaces. Or, in the context of creation of the universe from nothing [3], one considers all topologies with a given single boundary hypersurface.

The process of compactification is one in which the initial spacelike topology is $M=$ $S^{n-1}$ and the final topology is $M^{\prime}=S^{d-1} \times A$. An immediate consistency requirement is that there exists a manifold $X$ with boundaries $M$ and $M^{\prime}$. Consider filling in the $S^{n-1}$, to give the disc $D^{n}$. We now see that the question is the same as requiring the existence of a manifold $X$ with unique boundary $\partial X=M^{\prime}$, describing the creation from nothing of a compactified universe. Such issues are the concern of cobordism theory, which is introduced briefly below.

The manifold $X=D^{d} \times A$, where $D^{d}$ is the $d$-dimensional disc, clearly provides a manifold with boundary $S^{d-1} \times A$, as required. No internal manifold $A$ is ruled out at this level. However, for various reasons, one would like a more systematic understanding of compactification.

The most pressing concern arises in a causal Lorentzian approach to quantum gravity. Here, the intermediate manifold, or cobordism, $X$, is considered together with an almost Lorentzian metric that gives a well-defined causal structure on $X$. Well-known theorems forbid causal topology change with a fully Lorentzian metric $[4,1]$. Almost Lorentzian metrics evade these theorems by allowing the metric to be degenerate at certain points $[6,5,3]$. Whilst such almost Lorentzian metrics always exist, it is conjectured [7] that the structure only contributes to the path integral if it is causally continuous, as described below. It has been shown that the requirement of a causally continuous almost Lorentzian metric is equivalent to certain topological conditions on $X[8,9]$. Thus, in order for a given compactification to be possible, one needs to prove the existence of a manifold $X$ satisfying the required conditions. Generically, $X=D^{d} \times A$ will not satisfy these conditions.

Even outside the causal Lorentzian approach, one would like to know what kind of topologies are possible for $X$. Is there a 'simplest' allowed topology? Adding dynamics to the system at a later stage may impose further topological restrictions. We will obtain cobordisms $X^{\prime}$, with different topology to $X$, by surgery on $X$. The causal structure of $X^{\prime}$ is then studied using handle decomposition.

Surgery theory and handle decomposition have been used previously in discussions of topology change in the physics literature, see for example $[10,7,11,12,13]$. Previous notes on higher dimensions and topology change are $[1,14]$. However, many of the powerful 
theorems in these areas of mathematics were not used. We will see that some of these theorems are very useful for studying higher dimensional topology change.

One important result of this work is that any compactification $S^{d-1} \times A$ may be obtained via a manifold $X$ that admits a causally continuous Lorentzian metric, if the total dimension of spacetime $n \geq 5$. Thus, no compactification is ruled out at this level in causal Lorentzian quantum gravity. Indeed the result is more general, and any topology change in these dimensions, between connected initial and final topologies, that is possible on cobordism grounds may be achieved in a causally continuous way. This result was already known for $n=4$ spacetime dimensions [7].

Another important point is that when compactifying on group manifolds, and in general via parallelisable cobordisms, we will see that it is possible to systematically rearrange the cobordisms for the process of compactification.

Section 2 reviews mathematical results from relative homology and homotopy theory, surgery theory, handle decomposition and Morse theory. Section 3 shows how the topology of the cobordism for a generic compactification may be rearranged such that the resulting cobordism admits a causally continuous almost Lorentzian metric. Section 4 considers the case of compactification via parallelisable cobordisms, where significantly further rearrangement is possible. Section 5 contains remarks on when all cobordisms can be obtained via surgery on the initial cobordism $X$. Section 6 is a concluding discussion. The appendix contains an argument for why surgery is a natural operation to consider on a manifold.

\section{Mathematical preliminaries}

All manifolds discussed are assumed to be smooth, connected and compact.

\subsection{Results from relative homology and homotopy theory}

Let $A$ be a subspace of the topological space $X$, and $S_{k}(X)$ the free abelian group of kchains in $X$. Let $\partial_{k}: S_{k}(X) \rightarrow S_{k-1}(X)$ be the boundary operator. The group of relative k-cycles $\bmod A$ is

$$
Z_{k}(X, A)=\left\{\gamma \in S_{k}(X): \partial_{k} \gamma \in S_{k-1}(A)\right\}
$$

The group of relative k-boundaries $\bmod A$ is

$$
B_{k}(X, A)=\left\{\gamma \in S_{k}(X): \gamma-\gamma^{\prime} \in B_{k}(X), \text { some } \gamma^{\prime} \in S_{k}(A)\right\}
$$


where $B_{k}(X)$ is the usual group of k-boundaries. The relative homology groups are now defined as

$$
H_{k}(X, A)=Z_{k}(X, A) / B_{k}(X, A)
$$

We will be mostly interested in the case when $A=\partial X$, the boundary of $X$. The two following theorems from relative homology theory will be used. For extended discussions see e.g. $[15,16]$.

Theorem 1. Given a subspace $A$ of $X$, there exists an exact sequence

$$
\cdots \longrightarrow H_{k}(A) \longrightarrow H_{k}(X) \longrightarrow H_{k}(X, A) \longrightarrow H_{k-1}(A) \longrightarrow \cdots
$$

Theorem 2. (Lefschetz duality) Given an oriented manifold $X$ of dimension $n$ and boundary $\partial X$ then one has the isomorphisms $H^{k}(X) \cong H_{n-k}(X, \partial X)$ and $H^{k}(X, \partial X) \cong$ $H_{n-k}(X)$ for all $k$.

It can be useful to combine Lefschetz duality with the isomorphism of vector spaces $H^{k}(X) \cong H_{k}(X)$. This isomorphism only holds with coefficients in a field, it does not hold when there is torsion. However, the following theorem shows the precise effect of torsion $[15]$ :

Theorem 3. (Universal coefficient for cohomology) The sequence, with coefficients of the homology and cohomology groups shown explicitly,

$0 \longrightarrow \operatorname{Ext}_{\mathbb{Z}}^{1}\left(H_{k-1}(X, \mathbb{Z}), \mathbb{Z}\right) \longrightarrow H^{k}(X, \mathbb{Z}) \longrightarrow \operatorname{Hom}_{\mathbb{Z}}\left(H_{k}(X, \mathbb{Z}), \mathbb{Z}\right) \longrightarrow 0$, is exact (and splits).

All we need to know about the first term in the sequence, $\operatorname{Ext}_{\mathbb{Z}}^{1}\left(H_{k-1}(X, \mathbb{Z}), \mathbb{Z}\right)$, is that it gives the torsion of $H_{k-1}(X, \mathbb{Z})$. For the full definition, see for example [15]. The last term gives the non-torsion part of $H_{k}(X, \mathbb{Z})$. Thus, the theorem is saying that a copy of $\mathbb{Z}$ in $H_{k}(X, \mathbb{Z})$ contributes $\mathbb{Z}$ to $H^{k}(X, \mathbb{Z})$ and a copy of $\mathbb{Z}_{p}$ in $H_{k-1}(X, \mathbb{Z})$ contributes $\mathbb{Z}_{p}$ to $H^{k}(X, \mathbb{Z})$.

One also has relative homotopy spaces, $\pi_{k}(X, A)$. Most important will be $\pi_{1}(X, A)$, which is not a group and is given by the homotopy classes relative to $A$ of paths in $X$ that have one endpoint at a basepoint $x_{0} \in A$ and the other endpoint in $A$. The higher relative homotopy spaces are groups [15]. The following two theorems are important $[15,16]$ :

Theorem 4. Given a subspace $A$ of $X$, there exists an exact sequence

$$
\cdots \longrightarrow \pi_{k}(A) \longrightarrow \pi_{k}(X) \longrightarrow \pi_{k}(X, A) \longrightarrow \pi_{k-1}(A) \longrightarrow \cdots \longrightarrow \pi_{0}(X) .
$$

Theorem 5. (Hurewicz) If $\pi_{k}(X, A)=0$ for $1 \leq k \leq s-1$, then $H_{k}(X, A)=0$ for $1 \leq k \leq s-1$. If, further, $\pi_{1}(X)=\pi_{1}(A)=0$, then $\pi_{s}(X, A) \cong H_{s}(X, A)$. 


\subsection{Surgery theory and cobordism}

An oriented manifold $X$ is an oriented cobordism between the oriented manifolds $M$ and $M^{\prime}$ if $\partial X$, with the induced orientation, is diffeomorphic to the disjoint union of $M$ and $-M^{\prime}$. Here, - denotes orientation reversal. Cobordism defines an equivalence relation on the space of oriented manifolds. We will generally be interested in the case $M=\emptyset$. Thus the manifold $X$ has connected boundary $\partial X=M^{\prime}$, neglecting the change in orientation. There is a simple criterion for when two manifolds without boundary are oriented-cobordant $[17,18]$ :

Theorem 6. Let $M, M^{\prime}$ be manifolds without boundary. Then $M$ and $M^{\prime}$ are orientedcobordant if and only if they have the same Pontrjagin and Stiefel-Whitney numbers.

Given a cobordism $X$, it is possible to obtain a different cobordism $X^{\prime}$, with $\partial X=\partial X^{\prime}$, through surgery on $X$. Suppose $X$ is $n$ dimensional. Intuitively, surgery, also known as spherical modification, should be thought of as removing an embedded sphere of dimension $k$ and replacing it with an embedded sphere of dimension $n-k-1$. A more precise description is as follows [19, 20, 21].

Start with an embedding of $\phi: S^{k} \times D^{n-k} \rightarrow X$. The boundary of the embedding is $S^{k} \times S^{n-k-1}$, which is also the boundary of $D^{k+1} \times S^{n-k-1}$. We may thus remove the interior of the embedding and replace it with the interior of $D^{k+1} \times S^{n-k-1}$. The result is the manifold

$$
X^{\prime}=\left(X-\phi\left(S^{k} \times 0\right)\right)+\left(D^{k+1} \times S^{n-k-1}\right),
$$

where - denotes removal and + denotes an identification of $\phi(u, \theta v)$ with $(\theta u, v)$ for each $u \in S^{k}, v \in S^{n-k-1}$ and $0<\theta \leq 1$. This will be called a type $(k, n-k-1)$ surgery. The process is illustrated in Figure 1.

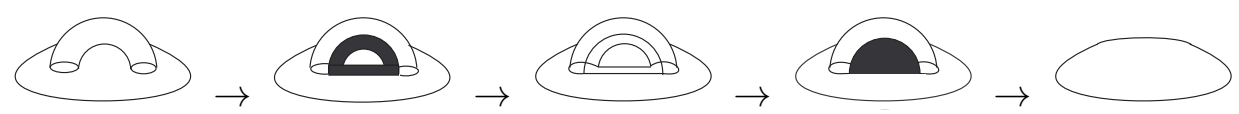

Figure 1: Surgery between $X$ and $X^{\prime}$, both with boundary $S^{1}$. An $S^{1} \times D^{1}$ is removed and replaced with a $D^{2} \times S^{0}$. The change in topology is evident.

Note that we are using surgery to modify the cobordism itself. This should not be confused with the use of surgery to construct cobordisms by modifying manifolds without boundaries. By performing surgeries below, we will find cobordisms for quantum compactification with desirable topological properties. 


\subsection{Handle decomposition, Morse theory and causal continuity}

Once we have obtained an interesting cobordism $X$, it will be useful to consider its handle decomposition $[22,21,10]$. A handle of index $k$ on an $n$ dimensional manifold, $X$, is an $n$-disc $D^{n}$ such that $X \cap D^{n} \subset \partial X$, and there is a homeomorphism $h: D^{k} \times D^{n-k} \rightarrow D^{n}$, such that $h\left(S^{k-1} \times D^{n-k}\right)=X \cap D^{n}$. Where $\partial D^{k}=S^{k-1}$. Two simple examples are shown in Figure 2. Adding a handle is closely related to performing a surgery, as we shall see below.

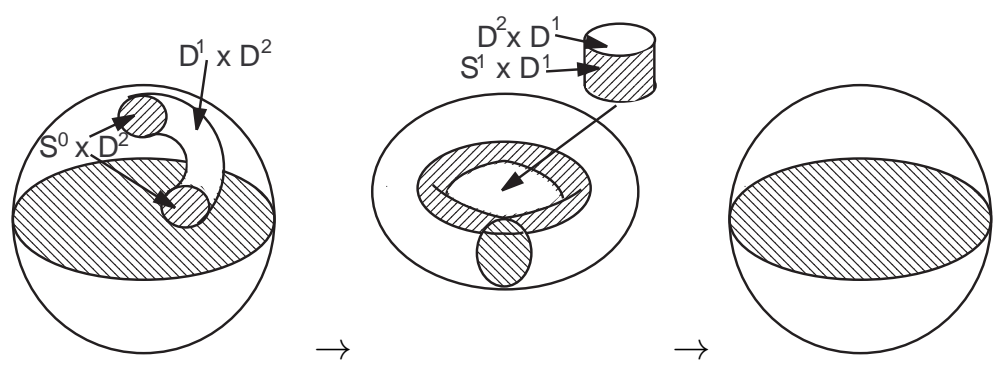

Figure 2: Adding a 1-handle to $D^{2}$ to obtain a solid torus. Adding a 2-handle to a solid torus to reobtain the ball.

A handle decomposition of a cobordism, $X$ from $M$ to $M^{\prime}$, is a presentation

$$
X=C_{0} \cup H_{1} \cup \cdots \cup H_{t},
$$

where $C_{0}=M \times[0,1]$ and $H_{k}$ is a handle on the cobordism

$$
X_{k-1}=C_{0} \cup\left\{\cup H_{l} \mid l \leq k-1\right\} .
$$

This gives a procedure for constructing $X$ from the trivial cobordism. If $\partial X$ has a single connected component, $M^{\prime}$, then one may start from the disc $C_{0}=D^{n}$. The handle decomposition of $X$ is not unique. For example, Figure 2 shows a handle decomposition of a 2-disc as a 2-disc with a 1-handle and a 2-handle added. Handle decompositions are generic by the following crucial result [22]:

Theorem 7. Every cobordism admits a handle decomposition .

Handle decomposition is also closely related to Morse theory [21, 23]. Morse theory will be used to define an almost Lorentzian metric on the cobordism with certain causal properties. A function $f: X \rightarrow \mathbb{R}$ has a critical point at $p \in X$ if $\partial_{i} f(p)=0$. The critical point is non-degenerate if $\operatorname{det}\left[\partial_{i} \partial_{j} f(p)\right] \neq 0$. A Morse function on a cobordism 
$X$ is a function $f: X \rightarrow \mathbb{R}$ that is constant on each connected component of $\partial X$ and whose critical points are in the interior of $X$ and non-degenerate. Every cobordism admits a Morse function, a result closely related to Theorem 7 .

The index of a non-degenerate critical point $p$ is the number of negative eigenvalues of the Hessian $\partial_{i} \partial_{j} f(p)$. The number of critical points with index $k$ will be denoted $\mu_{k}(f)$. The following result is important (this is theorem 3.12 of [21] translated into the language of handles):

Theorem 8. Given a handle decomposition of the cobordism $X$, then $X$ admits a Morse function with exactly one critical point of index $k$ for each $k$-handle in the decomposition.

The power of this result is that it gives us an equality for the number of critical points of a Morse function. This should be contrasted with the well-known weak Morse inequalities [23] $b_{k} \leq \mu_{k}(f)$, where $b_{k}$ are the Betti numbers of the manifold, $X$.

Given a Morse function $f$ on $X$ and a Riemannian metric $G$ on $X$, which always exists, one may then construct an almost Lorentzian metric [5]

$$
g_{\mu \nu}=G^{\rho \sigma} \partial_{\rho} f \partial_{\sigma} f G_{\mu \nu}-\zeta \partial_{\mu} f \partial_{\nu} f
$$

where $\zeta>1$ is a real number. This metric is Lorentzian everywhere except at the critical points and has a well-defined causal structure because $f$ acts as a time function. The timelike direction is $G^{\mu \nu} \partial_{\nu} f$. This almost Lorentzian metric is said to define a Morse spacetime.

The final idea we need is that of causal continuity [24]. Intuitively, a spacetime is causally discontinuous if the volume of the causal past or future of some point changes discontinuously under a continuous change in the point. A causally continuous spacetime may be characterised by the condition

$$
x \in \overline{I^{-}(y)} \Leftrightarrow y \in \overline{I^{+}(x)}, \quad \forall x, y \in X
$$

Where $\overline{I^{ \pm}(p)}$ denotes the closure of the chronological past and future of $p$. Recall that the chronological future of a point consists of all other points that may be reached via timelike curves. The closure in equation (8) is what makes the definition work.

It is conjectured [7] that causally discontinuous spacetimes do not contribute to the Lorentzian sum over histories. It was further conjectured [7] that causal continuity should be associated with critical points of index 1 and $n-1$ of Morse functions. It was later proven $[9,8]$ that: 
Theorem 9. If all Morse functions on a cobordism $X$ contain critical points of index 1 or $(n-1)$, then the cobordism supports only causally discontinuous Morse spacetimes. Conversely, if $X$ admits a Morse function with no critical points of index 1 or $(n-1)$, then it does support causally continuous Morse spacetimes.

Thus, putting the above results together, in the context of causal Lorentzian quantum gravity, there is a selection rule for topology change. Topology change requires a cobordism with a handle decomposition with no 1-handles or $(n-1)$-handles. We will see that this requirement is trivial in dimensions $n \geq 5$. It is already known in the physics literature to be trivial in dimension $n=4$ due to the Lickorish-Wallace theorem for three-manifolds [7].

\section{Quantum compactification: generic case}

In this section we will see that there exists a simply connected cobordism for the quantum creation of any compactified universe, $S^{d-1} \times A$, from nothing. We will then see that this cobordism admits a causally continuous almost Lorentzian metric.

An obvious cobordism for the creation of the compactified universe is $X=D^{d} \times A$, with boundary $S^{d-1} \times A$. From this starting point we will then perform surgery, if necessary, to obtain a simply connected cobordism $X^{\prime}$. Given $X^{\prime}$ one can then consider a handle decomposition of the cobordism and show that it admits a Morse function with no critical points of index 1 or $n-1$. The process is summarised as follows:

$$
D^{d} \times A \stackrel{\text { surgery }}{\longrightarrow} X^{\prime}, \pi_{1}\left(X^{\prime}\right)=0 \stackrel{\text { decomp. }}{\longrightarrow} f: X^{\prime} \rightarrow \mathbb{R}, \mu_{1}(f)=\mu_{n-1}(f)=0 .
$$

We will work in a more general framework, in which $X$ is a cobordism between $M$ and $M^{\prime}$. In the compactification case $M=\emptyset$ and $M^{\prime}=S^{d-1} \times A$.

\subsection{Eliminating the fundamental group}

The possibility of eliminating the fundamental group through surgery is contained in the following theorem due to Milnor [19] and Wallace [20]:

Theorem 10. An oriented compact manifold, with or without boundary and of dimension $n \geq 4$, can be made simply connected through a finite sequence of surgeries of type $(1, n-2)$.

The result of the surgeries, $X^{\prime}$, thus satisfies $H_{1}\left(X^{\prime}\right)=\pi_{1}\left(X^{\prime}\right)=0$. 
In fact a little more is true. Suppose $X$, hence $X^{\prime}$, is a cobordism between $M$ and $M^{\prime}$. Then we can now easily see that $\pi_{1}\left(X^{\prime}, M\right)=\pi_{1}\left(X^{\prime}, M^{\prime}\right)=0$. Consider the end of the exact sequence of Theorem 4 , given that $\pi_{1}\left(X^{\prime}\right)=0$ :

$$
0 \longrightarrow \pi_{1}\left(X^{\prime}, M^{\prime}\right) \longrightarrow \pi_{0}\left(M^{\prime}\right) \longrightarrow \pi_{0}\left(X^{\prime}\right)
$$

However, $\pi_{0}\left(M^{\prime}\right) \cong \pi_{0}\left(X^{\prime}\right)$, because $M^{\prime}$ and $X^{\prime}$ are connected and the inclusion map induces the isomorphism. Exactness then implies that $\pi_{1}\left(X^{\prime}, M^{\prime}\right)=0$. This result is also straightforward to see directly. If $M^{\prime}=\emptyset$ then we get the same result because $\pi_{1}\left(X^{\prime}, M^{\prime}\right)=$ $\pi_{1}\left(X^{\prime}\right)=0$. Clearly the same argument may be used for $M$ instead of $M^{\prime}$. The following subsection will further consider the cases when $X^{\prime}, M$ or $M^{\prime}$ are not connected.

In the appendix, a motivation is given for considering surgery as a 'minimal' transformation on manifolds that may eliminate homotopy groups.

\subsection{A causally continuous Morse metric for compactification}

We have obtained a cobordism for compactification, $X^{\prime}$, with $\pi_{1}\left(X^{\prime}\right)=\pi_{1}\left(X^{\prime}, M\right)=$ $\pi_{1}\left(X^{\prime}, M^{\prime}\right)=0$. This now allows us to find a particularly amenable handle decomposition of $X^{\prime}$. The important statement in this subsection is Theorem 12 below, but some preliminary remarks concerning connectivity, 0-handles and $n$-handles are needed.

First note that without loss of generality, we may assume that the initial cobordism $X$, and hence $X^{\prime}$, is connected. Suppose $X$ were not connected. Then we could make it connected by use of type $(0, n-1)$ surgeries. Concretely, in order to connect two components, remove a small disc from each, $S^{0} \times D^{n}$, and then join the components with $D^{1} \times S^{n-1}$. Once $X$ is connected, connectivity of $X^{\prime}$ follows because connectivity is not affected by the type $(1, n-2)$ surgeries of Theorem 10. In fact, we don't strictly need to do this, we could work with a disconnected cobordism by considering each connected component to give an independent cobordism, and then applying the argument below to each component separately. We may now use the following result [22]:

Lemma 11. If $X^{\prime}$ is a connected cobordism from $M$ to $M^{\prime}$, then $X^{\prime}$ admits a handle decomposition with no 0 -handles if $M \neq \emptyset$ and no $n$-handles if $M^{\prime} \neq \emptyset$.

The case $M=\emptyset$ or $M^{\prime}=\emptyset$ do not pose a problem for our purposes. In, say, the case $M=\emptyset$, remove a small disc $D^{n}$ from $X^{\prime}$. Now view $X^{\prime}$ as broken into two sequential cobordisms; the first, $D^{n}$, from $\emptyset$ to $S^{n-1}$, and the second, $X^{\prime}-D^{n}$, from $S^{n-1}$ to $M^{\prime}$. The first of these cobordisms is trivial, made up of a 0-handle and nothing else. The second 
cobordism now fulfills the criteria of Lemma 11. In the case where originally we had $X^{\prime}$ with $M=\emptyset$, we shall instead regard $X^{\prime}$ as being the second cobordism, with the $D^{n}$ excised, and therefore $M=S^{n-1}$. We will then simply add the $D^{n}$ back in after applying the following theorem $[22,21]$ :

Theorem 12. Suppose $X^{\prime}$ is a connected cobordism from $M$ to $M^{\prime}$ with a handle decomposition with no 0 -handles and $i_{k} k$-handles for $k>0$. Suppose $\pi_{1}\left(X^{\prime}, M\right)=0$ and $n \geq 5$. Then $X^{\prime}$ admits a different handle decomposition with $i_{k}$ handles for $k \neq 1,3$, no 1-handles and $\left(i_{1}+i_{3}\right)$ 3-handles.

Thus we may always find a handle decomposition for $X^{\prime}$ which has no 1-handles. Adding back in an excised $D^{n}$, if necessary, adds only a 0-handle to the decomposition, and no 1handles. Further, by considering the 'dual handle decomposition' [22], which views the cobordism in the opposite direction, from $M^{\prime}$ to $M$, Theorem 12 may further be used to remove the $(n-1)$-handles, because $\pi_{1}\left(X^{\prime}, M^{\prime}\right)=0$. By Theorems 8 and 9 we then see the cobordism $X^{\prime}$ admits a causally continuous almost Lorentzian metric. No compactification is ruled out due to causal discontinuities for dimensions $n \geq 5$.

More generally, we see that no topology change in higher dimensions, with connected $M$ and $M^{\prime}$, is ruled out through considerations of causal continuity. Some topology change will be ruled out on the grounds of Theorem 6. By constructing the initial cobordism $X$ explicitly above, we saw that this is never a problem for compactification. The condition of $n \geq 5$ arises from uses of the Whitney Lemma [22], which is used to rearrange handles so that the intersections of handles may be placed in a canonical form.

In the above we have assumed that the initial and final topologies, $M$ and $M^{\prime}$, are connected. Consider the case that $M^{\prime}$ has various disconnected components. The key step above that no longer holds is that following equation (9); if $X^{\prime}$ is connected, then $\pi_{0}\left(M^{\prime}\right)$ and $\pi_{0}\left(X^{\prime}\right)$ are no longer isomorphic. It follows from (9) that $\pi_{1}\left(X^{\prime}, M^{\prime}\right)$ is nonzero, contrary to the requirements of our argument for eliminating $(n-1)$-handles.

In order to solve this problem, we would need $X^{\prime}$ to be disconnected, with one component containing each component of $M^{\prime}$. This would restore the desired isomorphism $\pi_{0}\left(M^{\prime}\right) \cong$ $\pi_{0}\left(X^{\prime}\right)$. We could now think of each connected component of $X^{\prime}$ as a cobordism in itself and apply Theorem 12 to each component separately. Unfortunately, whilst we have seen above that any two cobordant manifolds are cobordant via a connected cobordism, it is not necessarily true that there will exist a disconnected cobordism with the properties that we have just required. In particular, suppose $M=\emptyset$ and $M^{\prime}=N \sqcup N^{\prime}$ has two components. Suppose further that neither $N$ nor $N^{\prime}$ are cobordant to the empty set, that is, they do not 
bound. Then there does not exist a cobordism with two components with boundaries $N$ and $N^{\prime}$ because, by assumption, $N$ and $N^{\prime}$ do not bound. For example, let $N=-N^{\prime}=\mathbb{C P}^{2}$. Here - denotes orientation reversal. If we wanted to work only with spin manifolds, then we should use spin cobordism rather than oriented cobordism. In this case an example, in eight spatial dimensions, would be $N=-N^{\prime}=\mathbb{H}^{2}$.

To summarise, our argument will not work in circumstances such as when $M=\emptyset$ and when $M^{\prime}$ is disconnected and with two or more components that do not individually bound. Thus, requiring causal continuity might forbid the simultaneous creation of multiple universes with topologies that do not bound.

\section{Compactification via parallelisable cobordisms}

In this section we see that when the cobordism, $X$, is a parallelisable manifold, then the topology for quantum compactification may be rearranged considerably further than in the generic case. Recall that Lie groups are parallelisable. Note that this class of spacetimes includes torus compactifications. Weaker conditions than parallelisability are possible [19], but will not be studied here.

\subsection{Eliminating higher homotopy groups}

For a generic manifold, eliminating the fundamental group was the only possibility. This is because in order to perform the surgeries that eliminate homotopy groups, $\pi_{k}(X)$, one needs to use embedded spheres with trivial normal bundle, in order to embed $S^{k} \times D^{n-k}$. For any orientable manifold, an embedded $S^{1}$ will have trivial normal bundle, but this will not be true for higher dimensional embedded spheres.

However, if the cobordism has additional properties, then it will be possible to find spheres representing homotopy classes that have trivial normal bundles. In this case one can use surgery to eliminate the homotopy class by collapsing the sphere.

Following $[19,25]$, define a manifold, $X$, to be $\mathbf{S}$-parallelisable if the sum $T(X) \oplus L$ is a trivial bundle. Here $T(X)$ is the tangent bundle and $L$ is a trivial line bundle over $X$. One now has the following lemmas [25]:

Lemma 13. If $X$ is a submanifold of $S^{n+m}, n<m$, then $X$ is S-parallelisable if and only if its normal bundle is trivial.

Lemma 14. A connected manifold with non-vacuous boundary is S-parallelisable if and only if it is parallelisable. 
However, it is an elementary fact that a Lie group is a parallelisable manifold. Further, $D^{d}$ is also clearly parallelisable and therefore one has:

Corollary 15. The cobordism $X=D^{d} \times A$ is S-parallelisable, for any Lie group $A$.

An example of a parallelisable manifold that is not a Lie group is $S^{7}$. The usefulness of S-parallelisability is that it allows us to eliminate many homotopy groups $[19,25,20]$ :

Theorem 16. Let $X$ be compact, connected, and S-parallelisable, of dimension $n \geq 2 m$. Then, by a sequence of surgeries, one can obtain an S-parallelisable manifold, $X^{\prime}$, with $\pi_{k}\left(X^{\prime}\right)=0$ for all $k \leq m-1$.

A consequence of this theorem is that the homology of $X^{\prime}$ is largely determined by that of the boundary $\partial X^{\prime}=\partial X$ using Theorems 2 and 3. Theorem 5 implies that $\pi_{k}\left(X^{\prime}\right)=$ $0,0<k \leq m-1 \Rightarrow H_{k}\left(X^{\prime}\right)=0,0<k \leq m-1$. One has, for even dimensions $n=2 m$,

$$
\begin{aligned}
& H_{0}\left(X^{\prime}\right)=\mathbb{Z}, \\
& H_{k}\left(X^{\prime}\right)=0 \quad 1 \leq k \leq m-1, \\
& H_{m}\left(X^{\prime}\right) \quad \text { not determined from boundary }, \\
& H_{m+k}\left(X^{\prime}\right)=H_{m+k}(\partial X) \quad 1 \leq k \leq m-2, \\
& H_{2 m-1}\left(X^{\prime}\right)= H_{2 m}\left(X^{\prime}\right)=0 .
\end{aligned}
$$

These results follow from Theorem 16 as an elementary exercise using Theorems 1, 2 and 3. The final line of (10) requires $\partial X^{\prime}$ to be connected. A similar list is possible in odd dimensions. The only difference is that if $n=2 m+1$, then both $H_{m}\left(X^{\prime}\right)$ and $H_{m+1}\left(X^{\prime}\right)$ are not determined by the boundary.

This elimination of homology groups is all we shall use below. Note however, that the vanishing of homology groups has implications for handle decompositions [22]:

Theorem 17. Let $X$ be a cobordism from $M$ to $M^{\prime}$ with no handles of index $<s$ and $i_{t} t$-handles for $t \geq s$. Then, if $M$ is simply connected and $2 \leq s \leq n-4, n \geq 6$ and $H_{s}(X, M)=0$, we can find a new handle decomposition with the same number of $t$-handles for $t \neq s, s+1$, with no $s$-handles and with $i_{s+1}-i_{s}(s+1)$-handles.

This theorem implies that for an S-parallelisable manifold one may find a handle decomposition on $X^{\prime}$ with no $k$-handles for $k \leq m-1$. Note that here $M=\emptyset$, so $H_{k}\left(X^{\prime}, M\right)=$ $H_{k}\left(X^{\prime}\right)=0$, for $k \leq m-1$ as required. Note that one cannot generically eliminate handles above the middle dimension through considering the dual decomposition, because this would require $H_{k}\left(X^{\prime}, M^{\prime}\right)$ to vanish. 
There are also various circumstances under which one can do even better than in the previous subsection and further eliminate the $m$ th homology group through surgery, where $n=2 m$ or $n=2 m+1$, in the even and odd dimensional cases respectively $[19,26]$. In this case the homology is entirely determined from the boundary. This will not be discussed further here.

To illustrate the results of this section, consider now two examples.

\subsection{Example: Torus compactification from six to four dimensions}

The initial cobordism is $X=D^{4} \times T^{2}$ with boundary $\partial X=S^{3} \times T^{2}$. Theorem 16, Lefschetz duality, and the universal coefficient theorem allow us to find a cobordism $X^{\prime}$ with the following homology known:

$$
\begin{array}{r}
H_{0}\left(X^{\prime}\right)=\mathbb{Z}, \\
H_{1}\left(X^{\prime}\right)=H_{2}\left(X^{\prime}\right)=H_{5}\left(X^{\prime}\right)=H_{6}\left(X^{\prime}\right)=0, \\
H_{4}\left(X^{\prime}\right)=H_{4}(\partial X)=\mathbb{Z} \oplus \mathbb{Z} .
\end{array}
$$

The remaining group, $H_{3}\left(X^{\prime}\right)$, is not given by the boundary topology. However, it cannot be zero because the exact sequence

$$
0 \longrightarrow H_{3}(\partial X) \longrightarrow H_{3}\left(X^{\prime}\right) \longrightarrow H_{3}\left(X^{\prime}, \partial X\right) \longrightarrow H_{2}(\partial X) \longrightarrow 0
$$

would then imply $H_{3}(\partial X)=0$, which is false. Thus $H_{3}\left(X^{\prime}\right) \neq 0$.

The absence of first and second homology groups in the cobordism $X^{\prime}$ is something that we could not have achieved by taking the other obvious cobordism $X^{\prime \prime}=S^{3} \times D^{2} \times S^{1}$ (which incidentally is also parallelisable, cf. Corollary 20 below). We can see that what we have done is trade the lower dimensional homology of $X, H_{1}(X)=\mathbb{Z} \oplus \mathbb{Z}, H_{2}(X)=\mathbb{Z}$, for the higher dimensional homology of $X^{\prime}$.

\subsection{Example: $S^{4} \times T^{3}$ compactification from eleven to four dimensions}

Consider the eleven dimensional cobordism $X=D^{5} \times S^{3} \times T^{3}$ with boundary $\partial X=$ $S^{4} \times S^{3} \times T^{3}$. The cobordism is parallelisable because $S^{3}=S U(2)$ is parallelisable.

This cobordism may be considered as either a compactification from eleven to five dimensions on $S^{3} \times T^{3}$, or as a compactification from eleven to four dimensions on $S^{4} \times T^{3}$. In the latter case, the internal manifold is not a Lie group, but this doesn't matter because we are filling in the $S^{4}$ to get the initial cobordism, not the 'noncompact' (i.e. large) $S^{3}$, which is a Lie group. 
The initial cobordism has six nonzero homology groups (not counting $H_{0}(X)$ ):

$$
\begin{array}{r}
H_{1}(X)=H_{2}(X)=H_{4}(X)=H_{5}(X)=\mathbb{Z} \oplus \mathbb{Z} \oplus \mathbb{Z}, \\
H_{3}(X)=\mathbb{Z} \oplus \mathbb{Z}, \\
H_{0}(X)=H_{6}(X)=\mathbb{Z} .
\end{array}
$$

After applying Theorem 16 we get a cobordism $X^{\prime}$ with five nonzero homology groups:

$$
\begin{array}{r}
H_{1}\left(X^{\prime}\right)=H_{2}\left(X^{\prime}\right)=H_{3}\left(X^{\prime}\right)=H_{4}\left(X^{\prime}\right)=H_{10}\left(X^{\prime}\right)=H_{11}\left(X^{\prime}\right)=0, \\
H_{5}\left(X^{\prime}\right), H_{6}\left(X^{\prime}\right) \neq 0, \\
H_{7}\left(X^{\prime}\right)=H_{7}(\partial X)=\mathbb{Z} \oplus \mathbb{Z}, \\
H_{8}\left(X^{\prime}\right)=H_{8}(\partial X)=\mathbb{Z} \oplus \mathbb{Z} \oplus \mathbb{Z}, \\
H_{9}\left(X^{\prime}\right)=H_{9}(\partial X)=\mathbb{Z} \oplus \mathbb{Z} \oplus \mathbb{Z} .
\end{array}
$$

The nonvanishing of $H_{6}\left(X^{\prime}\right)$ and $H_{5}\left(X^{\prime}\right)$ follows from the nonvanishing of $H_{6}(\partial X)$ and $H_{5}(\partial X)$ respectively using the exact sequence of Theorem 1 in a similar way to (12).

\section{Completeness of surgery for compactification}

What are the limitations of a surgical analysis given an initial cobordism $X$ ? One natural question to ask is the following: Suppose we have two cobordisms $X$ and $X^{\prime}$ with connected boundaries, $\partial X=\partial X^{\prime}$, can one get from $X$ to $X^{\prime}$ via surgery?

The answer to this question uses the relation between handle decomposition and surgery, alluded to in section 2 above. The relation starts with the trivial cobordism $X=M \times[0,1]$, where $M$ has dimension $n-1$. If one adds a $k$-handle to one of the boundaries of $X$, to obtain $X^{\prime}$ with boundaries $M$ and $M^{\prime}$, then $M^{\prime}$ is obtained from $M$ via a type $(k-1, n-k-1)$ surgery. Said differently, given a cobordism $X^{\prime}$ between $M$ and $M^{\prime}$, the sequence of handles in a handle decomposition of $X^{\prime}$ corresponds to a sequence of surgeries required to go from $M$ to $M^{\prime}$. Note that here we are using surgeries on the endpoints of the cobordism, not on the cobordism itself as we did previously. Theorem 7 then implies, as is proved directly in $[19,20]$, that:

Corollary 18. Two manifolds without boundary $M$ and $M^{\prime}$ are cobordant if and only if $M^{\prime}$ may be obtained from $M$ via a sequence of surgeries.

Thus for manifolds without boundary, the question of completeness of surgery reduces to whether $M$ and $M^{\prime}$ are cobordant. This question is answered in Theorem 6 . 
This result may be generalised to manifolds with boundary as follows. The question is when two manifolds with boundary, i.e. cobordisms, $X$ and $X^{\prime}$, with $\partial X=\partial X^{\prime}$, may be obtained from one another via surgery. We will say that $X$ and $X^{\prime}$ are cobordant with boundary if there exists a manifold $W$ such that $\partial W=X \cup X^{\prime} \cup(\partial X \times[0,1])$, where $X$ and $X^{\prime}$ are attached to $(\partial X, 0)$ and $(\partial X, 1)$ in the obvious way. This is illustrated in Figure 3 , using the two cobordisms of Figure 1. We are essentially considering a 'cobordism of cobordisms'. One can generalise this concept to the case when $\partial X \neq \partial X^{\prime}[22]$.

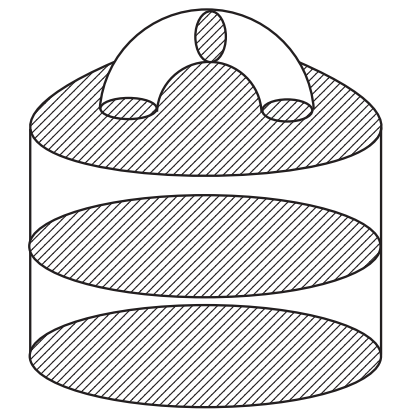

Figure 3: A cobordism with boundary. Here $\partial W=X \cup X^{\prime} \cup\left(S^{1} \times[0,1]\right)$.

Such a cobordism with boundary now admits a handle decomposition relative to $\partial X \times[0,1]$ in an entirely analogous way to the usual handle decomposition of a cobordism [22]. And, as we have just seen, this handle decomposition is equivalent to a series of surgeries. Thus one has:

Theorem 19. Two manifolds $X$ and $X^{\prime}$, with $\partial X=\partial X^{\prime}$, are related via surgeries if and only if there exists a manifold $W$ such that $\partial W=X \cup X^{\prime} \cup(\partial X \times[0,1])$.

Note that this contains within it the previous case where $\partial X=\emptyset$. This condition is more complicated than the case without boundaries. There are a couple of cases, however, in which we get a nice result. In dimensions 6 and 7, the oriented cobordism ring is trivial [18], implying that any manifold is cobordant to the empty set. In particular, this will be true for any $X \cup X^{\prime} \cup(\partial X \times[0,1])$ in these dimensions. Thus Theorem 19 implies:

Corollary 20. Any two cobordisms, $X, X^{\prime}$ with $\partial X=\partial X^{\prime}$, of dimension 6 or 7 are related via surgeries.

Therefore in these dimensions at least, we are not missing any cobordisms by restricting attention to those obtained from $D^{d} \times A$ by surgery. In other dimensions, cobordisms will fall into equivalence classes of those obtainable from one another via surgery [18]. 


\section{Conclusions and discussion}

This work has considered surgery as a canonical and systematic method of rearranging cobordisms for topology change. We have shown that any pair of connected cobordant manifolds in dimensions $\geq 5$ admits a causally continuous cobordism. This result extends the known result for 4 dimensions. A consequence is that quantum compactification is allowed in causal Lorentzian theories of quantum gravity, without selection rules.

We further illustrated the possibilities of surgical modifications by considering quantum compactification via parallelisable cobordisms.

Compactification is not the only higher dimensional topology change currently of interest. Recent results concerning instabilities in higher dimensions have hinted at the possibility of topology change $[27,28,29,30,31]$. More precisely, topology change arises in these works in two, related, ways ${ }^{1}$. Firstly, there seems to be a one-parameter family of solutions connecting compactified black strings and black hole spacetimes [28, 29]. Thus along this parameter the horizon topology, and also the singularity topology and the Euclidean spacetime topology, changes. Secondly, one considers the dynamical evolution of classical instabilities of uniform black string spacetimes [27] or generalised black hole spacetimes in which the instability is a 'ballooning' mode [30,31]. The perturbative dynamics, together with the lack of an obvious stable endpoint, suggest that the system is driven towards a regime in which quantum topology change may occur. In the black string case, this is related to the topology change along the one-parameter family of solutions mentioned previously. This is because the nonuniform black strings in the family of solutions have a higher mass than the unstable uniform black strings and the known lower mass solutions that could be available as endpoints of the instability are black holes and therefore have a different horizon topology.

\section{Acknowledgements}

I would like to thank Gary Gibbons and Burt Totaro for some very useful comments and references, and also James Sparks for some useful information. The author is funded by the Sims scholarship.

\footnotetext{
${ }^{1}$ I'd like to thank Toby Wiseman for a discussion on these points.
} 


\section{A Surgery as a canonical modification}

In this appendix we give one justification for considering surgery as a 'minimal' transformation of a manifold that eliminates the fundamental group. Suppose we have two manifolds $X=D^{d} \times A$ and $X^{\prime}$, not a priori related by surgery, with the same boundary. Further, we assume that $X^{\prime}$ is simply connected, whilst $X$ is not.

For simplicity, assume that both $X$ and $X^{\prime}$ have no torsion in their homology. This will enable us to assume an isomorphism between homology and cohomology. Consider the following pair of exact sequences, where Lefschetz duality, $H_{1}\left(X^{\prime}\right)=0$, and $H_{1}(X, \partial X)=$ $H_{n-1}(X)=H_{n-1}\left(D^{d} \times A\right)=H_{n-1}(A)=0$ have been used. Finally, $H_{1}(\partial X)=H_{1}\left(\partial X^{\prime}\right)$ is because $\partial X=\partial X^{\prime}$.

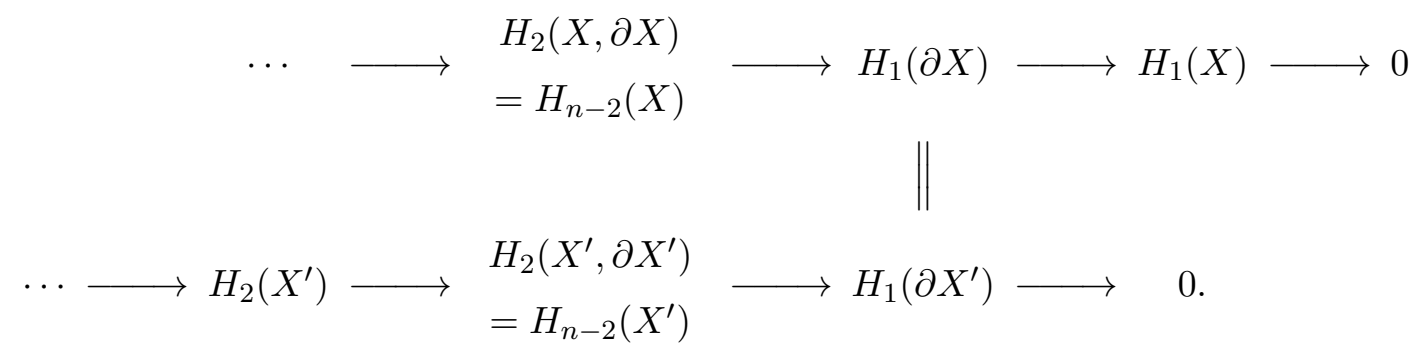

Suppose $H_{n-2}\left(X^{\prime}\right)=0$, this will lead us to a contradiction. Exactness of the second row would then imply that $H_{1}\left(\partial X^{\prime}\right)=H_{1}(\partial X)=0$. Now exactness of the first row implies that $H_{1}(X)=0$. But this contradicts the initial assumption that the starting cobordism $X$ was not simply connected. Thus we must have $H_{n-2}\left(X^{\prime}\right) \neq 0$.

The upshot of this argument is that any process eliminating the first homology from a manifold $X$ with $H_{n-1}(X)=0$ must necessarily introduce an $(n-2)$-th homology, if the boundary is preserved and modulo our torsion-free assumption. This is precisely what a surgery of type $(1, n-2)$ does in a minimalist way. The surgery collapses an $S^{1}$ and introduces an $S^{n-2}$. This is not a uniqueness proof, but gives some intuition for why surgery is natural in the present context. In fact, it is not difficult to give a precise meaning to the sense in which the change is minimal by proving that [20]:

Lemma 21. If a type $(k, n-k-1)$ surgery takes $X$ to $X^{\prime}$, then we have $H_{p}(X) \cong H_{p}\left(X^{\prime}\right)$ for all $p$ except $p=k, k+1, n-k-1, n-k$. 


\section{References}

[1] F. J. Tipler, "Topology Change In Kaluza-Klein And Superstring Theories," Phys. Lett. B 165 (1985) 67.

[2] S. W. Hawking, "Quantum Gravity And Path Integrals," Phys. Rev. D 18 (1978) 1747.

[3] A. Vilenkin, "Approaches To Quantum Cosmology," Phys. Rev. D 50 (1994) 2581 [arXiv:gr-qc/9403010].

[4] R.P. Geroch, "Topology in general relativity," J. Math. Phys. 8(4) (1967) 782.

[5] J. Louko and R. D. Sorkin, "Complex actions in two-dimensional topology change," Class. Quant. Grav. 14 (1997) 179 [arXiv:gr-qc/9511023].

[6] G. T. Horowitz, Class. Quant. Grav. 8 (1991) 587.

[7] F. Dowker and S. Surya, "Topology change and causal continuity," Phys. Rev. D 58 (1998) 124019 [arXiv:gr-qc/9711070].

[8] A. Borde, H. F. Dowker, R. S. Garcia, R. D. Sorkin and S. Surya, "Causal continuity in degenerate spacetimes," Class. Quant. Grav. 16 (1999) 3457 [arXiv:gr-qc/9901063].

[9] H. F. Dowker, R. S. Garcia and S. Surya, "Morse index and causal continuity: A criterion for topology change in quantum gravity," Class. Quant. Grav. 17 (2000) 697 [arXiv:gr-qc/9910034].

[10] H. F. Dowker and R. S. Garcia, "A handlebody calculus for topology change," Class. Quant. Grav. 15 (1998) 1859 [arXiv:gr-qc/9711042].

[11] R. Ionicioiu, "Building blocks for topology change in 3D," arXiv:gr-qc/9711069.

[12] L. J. Alty, "Building blocks for topology change," J. Math. Phys. 36 (1995) 3613.

[13] M. Y. Konstantinov and V. N. Melnikov, "Topological Transitions In The Theory Of Space-Time," Class. Quant. Grav. 3 (1986) 401.

[14] R. Ionicioiu, "Topology change from Kaluza-Klein dimensions," arXiv:gr-qc/9709057.

[15] J.J. Rotman, An introduction to algebraic topology, Springer-Verlag, New York, 1988.

[16] C.R.F. Maunder, Algebraic topology, Dover publications, New York, 1970. 
[17] J. Milnor, "A survey of cobordism theory", Enseign. Math. 8 (1962) 16.

[18] J.W. Milnor and J.D. Stasheff, Characteristic classes, Princeton University Press, New Jersey, 1974.

[19] J. Milnor, "A procedure for killing homotopy groups of differentiable manifolds", Proc. Sympos. Pure Math. Vol. III pp. 39-55, 1961.

[20] A.H. Wallace, "Modifications and cobounding manifolds", Canad. J. Math. 12 (1960) 503.

[21] J. Milnor, Lectures on the h-cobordism theorem, Princeton University Press, New Jersey, 1965.

[22] C.P. Rourke and B.J. Sanderson, Introduction to piecewise-linear topology, SpringerVerlag, Berlin, 1972.

[23] J. Milnor, Morse Theory, Princeton University Press, New Jersey, 1963.

[24] S.W. Hawking and R.K. Sachs, "Causally continuous spacetimes," Commun. math. Phys. 35 (1974) 287.

[25] M.A. Kervaire and J.W. Milnor, "Groups of homotopy spheres: I", Ann. of Math. 77 (1963) 504.

[26] C.T.C. Wall, "Killing the middle homotopy groups of odd dimensional manifolds", Trans. Amer. Math. Soc. 103 (1962) 421.

[27] R. Gregory and R. Laflamme, "Black Strings And P-Branes Are Unstable," Phys. Rev. Lett. 70 (1993) 2837 [arXiv:hep-th/9301052].

[28] B. Kol, "Topology change in general relativity and the black-hole black-string transition," arXiv:hep-th/0206220.

[29] T. Wiseman, "From black strings to black holes," arXiv:hep-th/0211028.

[30] G. Gibbons and S. A. Hartnoll, "A gravitational instability in higher dimensions," Phys. Rev. D 66 (2002) 064024 [arXiv:hep-th/0206202].

[31] G. W. Gibbons, S. A. Hartnoll and C. N. Pope, "Bohm and Einstein-Sasaki metrics, black holes and cosmological event horizons," arXiv:hep-th/0208031. 